\title{
ÊTRE ENSEMBLE, ÉCOUTER, ÉVALUER LES MUSIQUES DU MONDE EN FESTIVAL
}

\begin{abstract}
A
u sein du monde foisonnant et militant des musiques du monde a cours une formule ambiguë : "l'écoute du monde", reliant la perception d'ailleurs musicaux et un souci d'ordre éthique, politique ou spirituel pour les hommes et sociétés peuplant ces contrées.
\end{abstract}

Des collectes ethnomusicologiques aux métissages de la world music, des mouvements de résistance à la défense de la cause multiculturelle, des projets militants d'éducation populaire à la quête de spiritualité, cette idée d'un lien étroit entre l'écoute de musique et ce souci des autres a suscité des mobilisations plurielles et contradictoires qui se sont exprimées de façon non exclusive, mais particulièrement saillante, dans le champ des musiques du monde.

Sur cette catégorie, la littérature scientifique a suggéré des points de vue tout aussi contrastés : fabrication d'altérités (Guilbault, 1993), exploitation des répertoires traditionnels par des auteurs-compositeurs occidentaux (Zemp, 1995), moteur de rencontres interculturelles (Bohlman, 2002 ; Aubert, 2003 ; White, 2013) et de pratiques pédagogiques (Laborde, 1998), les musiques du monde ont notamment intéressé les chercheurs pour l'imaginaire qu'elles véhiculent et les grands partages qu'elles contribuent à réactiver. Soulignant la prégnance de facteurs non musicaux - politiques, économiques, sociaux, identitaires -, ces études ont tendu à se centrer sur les discours entourant la production et la réception des musiques du monde plutôt que sur l'analyse de situations. Inversement, alors que le thème de l'écoute a suscité une abondante littérature ethnomusicologique, celleci se centre surtout sur les contextes d'origine de certains répertoires musicaux et chorégraphiques alors que leur circulation dans le champ des musiques du monde y est envisagée à la 
marge comme le symptôme d'une décontextualisation induisant une transformation des répertoires et des décalages d'appréciation (Aubert, 2003 ; Coll., 2001 ; Olivier, 2014). Plus récemment, des travaux sur des festivals - eux-mêmes produits sur la base de méthodes plurielles - ont permis de montrer l'articulation entre un dispositif et les pratiques d'amateurs (Maisonneuve, 2009), la pluralité des profils d'auditeurs de musiques du monde (Négrier, 2014), le décalage entre un imaginaire cosmopolite et la composition réelle des publics (Djakouane, 2014), sans toutefois aborder de front la question du lien social mis en œuvre dans ces événements. Comment des auditeurs réunis dans un festival de musiques du monde écoutent-ils ensemble de la musique?

Tout en puisant dans ces perspectives, les enquêtes que j'ai menées sur des festivals en France et en Allemagne ${ }^{1}$ m'ont permis de remettre en chantier cette question, en adoptant une approche situationnelle (Fornel \& Quéré, 1999) et pragmatique (Dodier, 1993). J'ai envisagé ces événements comme des "épreuves » permettant de questionner "l'articulation entre les attentes et dispositions engagées dans l'action et les expériences » des divers participants (Pecqueux \& Roueff, 2009) et comme des expériences publiques mettant en jeu, à travers la musique, des questionnements relatifs au vivre-ensemble (Cefaï \& Terzi 2012; Fabiani, 2012). Appliquée au questionnement de ce numéro, cette démarche me permet ici de réfléchir à l'articulation entre différentes dimensions de l'agir collectif imbriquées dans un événement tel qu'un festival de musiques du monde. Un tel événement juxtapose d'une part, et de manière assez constante, plusieurs représentations de "l'être ensemble " : un imaginaire de la diversité culturelle - où être ensemble signifie se retrouver entre personnes de différentes cultures -, des idéaux et valeurs associés au partage d'un moment de musique - où être ensemble signifie être réunis entre amateurs de musique partageant des attentes, dispositions et codes de comportement communs -, un questionnement d'ordre politique sur le vivre-ensemble - où être ensemble signifie être réunis entre citoyens d'une même société. Mais un concert de musiques du monde juxtapose aussi, d'autre part, des activités et formes d'interaction plurielles - l'écoute de musique, la lecture de programmes, la conversation, la consommation de boissons, entre autres. Décrire l'écoute dans cette perspective suppose donc de tenir compte de cette complexité qui fait que se superposent différentes modalités de "l'êtreensemble " et du "faire-ensemble ". Cela suppose en outre de ne pas se centrer sur le seul moment du concert, mais aussi de prendre en compte ce qui, en amont, contribue à informer une certaine attention à la musique. C'est à cette condition que j'entends ici 
interroger, au-delà d'une opposition entre "discours " et " pratiques ", l'articulation entre les attentes que génère un festival, le contexte dans lequel il s'insère et les modalités concrètes d'organisation d'une telle expérience collective.

Pour ce faire, je partirai d'un cas sur lequel j'ai effectué une enquête au long cours, entre 2006 et 2011 : les compétitions Creole, un "prix pour les musiques du monde d'Allemagne ${ }^{2}$ " qui donne lieu depuis 2006 à des festivals régionaux dans différentes localités d'Allemagne et à une finale bi-annuelle. Enquêtant sur Creole, je me suis impliquée en tant qu'auditrice et amatrice de musique, en tant qu'experte sollicitée pour rédiger des rapports et participer à des réunions et débats publics, et en tant que membre du jury d'une session régionale. J'ai aussi été amenée à dialoguer avec des personnes dont je n'ai pas directement occupé les places mais dont je me suis efforcée de saisir les raisons d'agir (Favret-Saada, 1977). Cette démarche a une conséquence sur le choix des acteurs que je décris ici comme participant à l'activité d'écoute : on verra s'exprimer non seulement les "simples spectateurs", mais aussi certaines personnes impliquées dans la réalisation du festival. Ce choix tient en partie à une particularité des festivals Creole, qui réunissent dans leur public une proportion importante de professionnels du monde de la musique. Il est aussi, au-delà de ce cas, un moyen de mettre en question une division établie des tâches parmi les chercheurs, qui conduit à étudier séparément les divers aspects de la production - musicale, logistique, économique, politique - et de la réception - recensions dans les médias, pratiques des amateurs, composition des publics - et ne permet du coup pas de saisir l'articulation entre les divers rôles et places que peut parfois endosser une même personne - en tant que musicien, organisateur, membre du jury, spectateur. Le fait d'écouter ensemble est en somme ici mis au regard d'un questionnement plus vaste sur le festival comme fait social, comme occasion de "faire ensemble "impliquant des modalités d'engagement différenciées.

Je commencerai pour cela par porter mon attention sur le processus de fabrique des festivals afin de montrer comment s'articulent, dans le cas de Creole, un imaginaire suffisamment vague pour s'appliquer à tous les festivals de musiques du monde et des attentes plus spécifiques que nourrit ce festival de par son inscription dans un contexte singulier. Qu'est-ce qu'écouter le monde dans le cadre des festivals Creole et comment se fabrique un tel moment de musique? Puis j'en viendrai aux concerts, en portant mon attention sur les cadres enchâssés dans ces situations et sur les tensions qui peuvent en résulter pour certains auditeurs. La mobilité et la liberté d'appropriation de ceux-ci font alors émerger 
ce questionnement : qu'est-ce qui est réellement partagé dans ce moment d'écoute collective? Enfin, je me pencherai sur la délicate production des verdicts au sein des jurys. Ce parcours mène en somme du registre de valeurs et attentes partagées à la description de situations publiques et intimes - concerts, délibérations -, requérant à chaque étape de spécifier ce qu' être ensemble » et " faire ensemble " veulent dire.

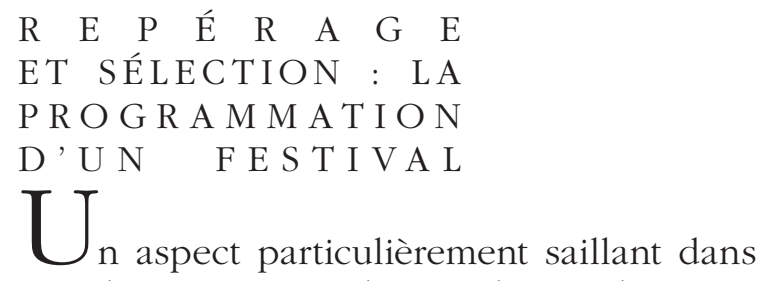

les discours entourant les "musiques du monde " est la récurrence d'un imaginaire associé à l'exploration. Cet imaginaire dessine la figure d'un auditeur idéal disposé, ainsi que nous y invitent les guides spécialisés (Bensignor, 2004; Broughton \& Burton, 1994), à ouvrir son oreille aux sonorités de tous les continents et à porter son attention, au-delà de ces sonorités, sur les personnes qui peuplent ces contrées et aux causes qu'ils défendent. Cet imaginaire est suffisamment indéfini pour alimenter des démarches multiples - d'enquêtes, de reportages et récits de voyage, de création, d'action sociale - qui ont pour dénominateur commun la conviction que, pour reprendre une formule attribuée à Gilbert Rouget, "la musique est toujours beaucoup plus que la musique ${ }^{3}$. Elle est aussi une voie d'accès à des cultures étrangères, à des minorités et des mouvements de résistance à l'oppression, aussi bien qu'à des spiritualités ou à des modes de vie alternatifs.

Autant cet imaginaire est consensuel et flexible, autant les choses se précisent pourtant dès lors que l'on se penche sur un festival en particulier ${ }^{4}$. C'est en suivant le processus complexe de repérage et de sélection qui s'effectue en amont des concerts que l'on peut parvenir à rendre compte de la spécificité de ces horizons d'attentes. Dans le cas des compétitions Creole, qui sont prises en charge par un collectif de plusieurs structures - des associations, des centres socioculturels, des agences privées, des administrations municipales -, l'objectif est de donner à voir et à entendre les « musiques du monde d'Allemagne » désignées par un concept emprunté au champ de la linguistique :

"Lorsque la combinaison de plusieurs langues en fait jaillir une nouvelle, on parle de langue "créole". Voilà précisément ce qui se passe aujourd'hui dans le monde de la musique, en Allemagne

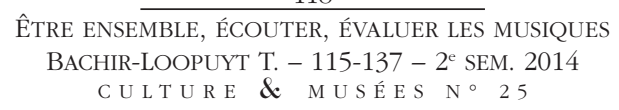


comme en Europe. De plus en plus de mondes musicaux se rencontrent. Le contexte de la globalisation amène aussi un élargissement constant des possibilités culturelles. " (Texte d'introduction au programme du prix Creole 2007)

Alors qu'une représentation commune des musiques du monde les associe à des ailleurs lointains, les compétitions Creole opèrent une inversion qui consiste à les réinscrire dans le local, non sans générer une certaine ambiguïté puisque les "musiques du monde d'Allemagne " peuvent être comprises à la fois comme des musiques d'origine étrangère fabriquées en Allemagne et comme des musiques originaires de certaines localités allemandes. Elles peuvent par ailleurs relever de différents genres ou mondes musicaux, ainsi qu'il est spécifié dans l'appel à candidatures : des " musiques traditionnelles " aux " musiques pop, jazz, musique contemporaine ", "cette compétition s'adresse à tous les groupes qui mettent en ouvre dans leur musique une rencontre interculturelle, un dépassement des frontières et des liaisons transculturelles".

Le spectre des attentes est donc large par rapport à d'autres festivals qui suivent des lignes de programmation plus restreintes. Mais il importe cependant que les candidats satisfassent à deux critères déterminants. D’une part, ils doivent se "référer de manière visible et explicite à une ou plusieurs cultures musicales régionales ou étrangères" (ibid.) : que ces musiques viennent d'ici ou d'ailleurs, il faut que l'on puisse voir et entendre - qu'elles viennent de quelque part, non seulement au sens du lieu où elles sont produites - l'Allemagne - mais aussi au sens d'une origine perceptible. D'autre part, ils ne doivent pas relever de certaines catégories : les "solistes, big band et chœurs", les ensembles de "musique classique", de "musique pop occidentale ou de musique contemporaine sans lien avec des éléments spécifiques à certaines cultures régionales ou étrangères " (ibid.).

Même une fois formulées ces limites, elles s'avèrent cependant délicates à poser en pratique. C'est ce que montre la phase suivante de l'appel à candidatures, qui consiste en une prospection menée dans les différentes villes et régions où se tient la compétition, pour laquelle les organisateurs des différentes sessions régionales sollicitent de nombreux experts locaux chargés de mission, musiciens, professionnels du monde de la musique, ethnomusicologues. L'enjeu, à ce stade, n'est pas de légiférer sur ce que doivent être les «musiques du monde d'Allemagne " mais de constituer un vivier de candidats, le plus large possible, sur la base de catégories éminemment flexibles. Vient ensuite une première sélection que les organisateurs 
confient à un comité de trois experts chargés de comparer les dossiers et $\mathrm{CD}$ de démonstration des candidats en fonction de trois critères - l'originalité, la qualité des compositions, la qualité de l'interprétation - et de sélectionner ceux qui seront programmés dans un festival régional.

Ces experts - qui peuvent être musiciens, journalistes, chercheurs, producteurs - sont choisis pour leur connaissance de certains répertoires mais aussi pour leur expérience dans le champ des musiques du monde, qui leur permet de prendre en compte, outre les critères officiels, des attentes partagées vis-àvis d'un festival de musiques du monde - l'attrait pour des ailleurs musicaux, la représentation de différentes aires culturelles, tendances ou genres musicaux. Il importe aussi qu'ils connaissent bien le contexte de cette compétition et de chaque région - Berlin et Brandenbourg, Rhénanie-Westphalie, Bavière, etc. ${ }^{5}$ - pour tenir compte de la spécificité du paysage musical régional et de la prégnance d'enjeux de société plus larges : entre autres, la question très débattue en Allemagne du multiculturalisme, qui fait que l'on entend ici aussi montrer la présence de certaines minorités issues de l'immigration, ou encore celle des rapports de genre, qui rend certains jurés attentifs à la proportion de femmes.

Au terme de cette prospection et de ces sélections, effectuées par des personnes différentes d'une région à l'autre, les festivals Creole en viennent ainsi à rassembler un ensemble composite de pratiques dont il importe peu que les musiciens les définissent eux-mêmes comme "créoles " ou comme " musiques du monde": musiques savantes et populaires d'Italie du Sud (Maramme), musiques à danser de Rhénanie (Einstrürzende Heuschober), hard rock turc (Safkan), musique contemporaine chinoise (Seidenstrasse), chanson hip-hop d'Amérique du Sud (Chupacabras), chants rituels soufis (Rumi Ensemble), mélange de jazz expérimental et de chants traditionnels d'Ukraine (Borderland), et bien d'autres encore. Avec ce paradoxe que ces musiques rassemblées dans une même compétition sont, dans le même mouvement, mises en concurrence : des quelque 500 ensembles candidats (en 2006-2007), seuls trois seront élus à la fin lauréats du prix Creole.

Sans qu'il soit ici question de passer en revue chaque étape de la fabrique des festivals Creole, voici ce que nous pouvons en retenir pour notre questionnement. Tout d'abord, cette manifestation repose sur un horizon d'attention multidimensionnel. Elle vise en premier lieu la musique mais aussi, à travers cette entrée, des questionnements qui ont cours dans la sphère publique : quant à la place et au rôle des musiques du monde, quant à la situation des arts dans un monde globalisé, quant au 
vivre-ensemble dans une société multiculturelle. Écouter le monde dans le cadre des festivals Creole, c'est porter son attention à la fois sur des réalités musicales plurielles - d'une région et d'un monde de musique à l'autre - et sur ces problèmes publics. Par ailleurs, la mise en ouvre collective du festival suppose de prendre en compte, au-delà de ces attentes, l'impact d'un arrière-plan institutionnel : une structuration fédérale de l'action culturelle - qui fait qu'à la différence d'un concours français, il apparaît ici normal de confier l'évaluation à des jurys différents d'une région à l'autre -, l'inscription des musiques du monde dans un champ de l'action socioculturelle - qui fait que les festivals sont généralement financés par des fonds dédiés à la Soziokultur plutôt qu'à la musique -, la prégnance de certaines catégories établies - qui fait que l'on considèrera comme plus plausible la participation de tel ou tel groupe. Chaque aspect du processus de fabrique d'un festival suppose de s'ajuster à des cadres et ressources variables (Bachir-Loopuyt, 2013), et ces multiples décrochages ont pour conséquence une incertitude d'autant plus grande sur le plan des objets et personnes visés par ce rassemblement.

Or, cette complexité du processus de fabrique des festivals a aussi un impact sur les expériences au moment des concerts et sur la manière dont j'entends à présent les décrire. Plutôt que de viser la caractérisation d'un type d'événement - le festival de musiques du monde - ou de publics, je voudrais ici montrer comment s'articulent, dans une même situation, différentes attentes et dispositions à agir ensemble.

\section{ÉCOUTER AVANT TOUT ? LE MOMENT DU CONCERT}

Vis-à-vis d'autres situations d'écoute, une des particularités du concert tient d'abord à la coprésence de différents acteurs qui partagent ensemble un moment de musique. On y retrouvera la plupart de ceux qui participent à la réalisation d'un festival - organisateurs, musiciens, techniciens, sponsors - ainsi que d'autres personnes - journalistes, invités, "simples spectateurs " - qui s'estiment concernées par celui-ci. Dans le cas de Creole, la configuration est la suivante : les concerts ont lieu dans des salles pouvant accueillir jusqu'à 500 spectateurs - 1500 pour les finales - et rassemblent une majorité de locaux - personnes impliquées dans l'organisation ou dans les institutions partenaires, amis des musiciens, professionnels et amateurs habitant la ville où se tient le festival ${ }^{6}$. Par ailleurs, ils présentent, ainsi que c'est couramment le cas dans un festival, une pluralité de prestations musiciennes : les festivals Creole ont ainsi lieu sur deux à quatre soirées, chacune faisant se succéder cinq à sept groupes de musique. 
Pour décrire ces occasions de musique, je prendrai ici comme entrée la notion de "cadres de l'expérience "(Goffman, 1991). Creole est une compétition. Cet aspect implique une insistance sur l'égalisation des formats de présentation de la musique - les concerts ont lieu sur une seule et même scène, chaque groupe candidat doit jouer 20 minutes - et contribue aussi à orienter les expériences des auditeurs vers une fin : le moment solennel de la remise des prix. Creole est aussi un festival, une fête qui vise à créer un moment d'effervescence collective en rassemblant une communauté de spectateurs autour de héros locaux. Creole est un concert, organisant la rencontre entre des musiques du monde et l'oreille de spectateurs. Creole est enfin une occasion de rencontre pour un réseau de professionnels ${ }^{7}$, et un tremplin pour des musiciens peu connus.

Ces différents principes de cadrages concourent cependant à définir un cadre d'interaction primaire dirigé vers l'écoute de musique. Le dispositif scénique, la disposition de la salle, la sonorisation et les éclairages, les programmes distribués à l'entrée, les présentations des groupes faites par un modérateur - ou, le plus souvent, une modératrice - contribuent à diriger prioritairement l'attention des auditeurs vers la musique. Mais il arrive cependant que certains incidents ou éléments parasites remettent en question, pour certains auditeurs, cette primauté de l'écoute. Un tel trouble a par exemple surgi à l'occasion de la seconde finale de Creole, pour laquelle les organisateurs avaient opté pour un lieu de concert plus grand que d'ordinaire : le Huxleys Neue Welt, une grande halle de $1225 \mathrm{~m}^{2}$ pouvant accueillir jusqu'à 1600 spectateurs. Le lendemain de la première soirée de concert, un débat qui se tenait à l'Atelier des Cultures sur les prix de musiques du monde glisse sur la question de l'écoute, à la suite d'une prise de parole de la chanteuse Urna Chahar-Tugchi, membre du jury de cette finale :

Urna Chahar-Tugchi : "J'entends tellement de choses électroniques et je me demande : mais pourquoi ? À quoi cela tient-il? J'ai pourtant fait l'expérience que les gens sont très attirés par la musique acoustique. Alors qu'ici, le son est fort de manière incessante. Je suis désolée de devoir dire cela, mais c'est la vérité ! Je sors d'un concert et je me pose ce genre de questions plutôt que : "Était-ce beau?" "

Un auditeur de la salle demande la parole (Maik Wolter, directeur de l'association Pro Folk) : "Je me pose moi aussi des questions sur la situation de présentation. Lorsque je suis rentré hier dans la salle, l'endroit le plus clair n'était pas la scène mais le comptoir du bar! Au beau milieu d'un concert à l'ambiance musicale intime, on entend le bruit des caisses de 
bière... Cela ne va pas du tout ! [...] Nous devons en revenir à cette question : qu'est-ce que la musique? De quoi les musiciens ont-ils besoin? Et déjà : combien de bons techniciens avons-nous?... »

Ce débat fait ressortir une dimension rarement thématisée dans les médias et débats publics, qui reste d'ordinaire cantonnée aux "Sanctions diffuses ${ }^{8}$ " ou pensées non exprimées 9 par les auditeurs : la question du son, de sa perception et appréciation qui tient à divers aspects - l'usage de l'amplification (électroniques vs acoustiques), le volume et la qualité du mixage, la configuration des lieux, mais aussi des différences entre des genres musicaux et entre ceux que l'on imagine être les amateurs de tel ou tel genre :

Maik Wolter : "Combien de bons techniciens avons-nous?" Un autre auditeur du public intervient (Christoph Sure, organisateur de Creole Basse-Saxe \& Brême) : «Là, Maik, je dois t'interrompre! Dans cette grande halle, j'ai pensé au départ que cela n'allait pas fonctionner. Mais finalement, j'ai très rarement entendu un son aussi bon et différencié. Bien sûr que c'est parfois fort, mais c'est la musique!»

Maik Wolter : "Mon problème n'est pas le volume..."

Le modérateur (Christian Rath) : "Avant que nous n'entrions dans une discussion d'experts (rires dans la salle), je dois préciser, pour ceux qui ne le connaissent pas, que Maik Wolter est président de la fédération Pro Folk. Il s'occupe donc de musiques plutôt douces...»

Maik Wolter : "C'est un préjugé sur le folk! Le folk est folklorique mais pas particulièrement doux. Vous avez déjà entendu une cornemuse? (rires dans la salle)»

Ce débat met en jeu des conceptions plurielles de la musique et de son écoute. Pour la première auditrice, les conditions de l'écoute sont perturbées par le volume trop fort ; pour le second, par des éléments parasites - bruits de voix ou de bouteilles, éclairages, animations visuelles - qui ne posent toutefois pas de difficulté à d'autres. Mais en filigrane de ce débat, il y a aussi la question de savoir si des musiques du monde peuvent être écoutées de la même manière que les formes présentées, par exemple, dans un concert de musique classique : soit, ainsi que l'on conçoit ordinairement cette forme d'écoute, en restant assis et en écoutant de manière concentrée, sans se livrer à une autre activité (boire, danser, etc.). C'est bien cette idée de l'écoute comme d'une "conduite esthétique" (Schaeffer, 1996) que les organisateurs de cette finale de Creole ont décidé d'entériner en disposant dans la salle du Huxleys Neue Welt des estrades pour permettre à 
certains spectateurs de s'asseoir et sur laquelle ils insistent encore lors de la finale suivante (Creole 2011) en prenant deux nouvelles mesures destinées à " discipliner " les auditeurs : désormais, la terrasse et le bar sont fermés pendant les concerts.

Ce faisant, ils risquent pourtant de contrecarrer une autre attente. L'ambiance n'est-elle pas devenue trop sérieuse, se demandera le journaliste Christian Rath (Folker) ? "Ce n'est pas drôle, personne ne danse ! ", s'exclamait déjà l'ethnomusicologue Raimund Vogels lors de la finale de Creole 2009, si différente selon lui de l'ambiance des sessions régionales de Creole en Basse-Saxe. Ces commentaires font ressortir des décalages entre ce que l'on attend d'un festival de musiques du monde et les expériences effectives : décalages rapportés à l'ambiance - trop "sérieuse" - mais aussi, parfois, à la composition du public - trop vieux ${ }^{10}$, trop " allemand"? - et aux conduites des spectateurs - "pourquoi ne dansent-ils pas? ». Ces décalages, cependant, ne sont pas constants et ils ne sont ressentis comme tels que par certains auditeurs - ceux qui estiment qu'un festival doit être nécessairement festif et qu'il faudrait y danser ${ }^{11}$. En outre, ils n'empêchent pas que les mêmes spectateurs puissent alterner, au cours d'une même soirée de concert, entre différentes activités et conduites d'écoute.

\section{P E R F O R M A N C E S M U S I C I E N N S E T CONDUITES D'ÉCOUTE \\ D \\ ans un festival tel que Creole se succè-} dent des performances qui impliquent des manières d'écouter et d'interagir très différentes. Prenons pour exemple la première soirée de la finale du prix Creole 2007, qui se tenait dans la salle du club du Domicil à Dortmund et lors de laquelle se sont succédé : un spectacle de théâtre musical d'Italie (Maramme), un quatuor de femmes a capella (Niniwe), un trio de chanson turque (Iki Dünya), un ensemble de musique contemporaine mongole (Egschiglen), une séquence de gnawa trance dance (El Houssaine Kili), un groupe de polkarock chantant en allemand (Hiss) et, pour finir dans une ambiance festive, un ensemble de reggae-soul-ska (Jamaram). Chacune de ces performances mise sur certains effets. Certaines se prêtent à la danse, d'autres non. Chacune implique une certaine conception de la "beauté " de la musique ou de sa vertu à relier différentes personnes (Bachir-Loopuyt, 2013 : 428-500).

Si l'on s'attache à présent à recenser certains faits et gestes des spectateurs, on observera par ailleurs une certaine mobilité dans 
l'espace et dans l'attention : les spectateurs peuvent changer de place, circuler entre différents lieux - la salle, le bar, les couloirs, le balcon, l'entrée -, adopter différentes postures - assis ou debout, en dansant, en chantant, sans bouger - et être plus ou moins attentifs à la musique (Pecqueux, 2003). Cette mobilité est toutefois tempérée par un répertoire de sanctions codifiées (applaudissements, encouragements, sifflets, ovations) et un principe de solidarité qui incite à encourager tous les musiciens, ce d'autant plus qu'ils participent à cette compétition bénévolement ${ }^{12}$. De manière systématique, les spectateurs des festivals Creole applaudissent donc à la fin de chaque morceau, ne huent jamais les groupes et restent le plus souvent en grande majorité dans la salle. Cette relative constance est cependant, il faut bien le dire, permise par le format des 20 minutes qui est assez court pour permettre de se plonger dans une variété de styles, y compris ceux que l'on n'apprécie pas. C'est à cette condition que les auditeurs peuvent se livrer à une écoute "omnivore" (Peterson, 2004).

Pour chacune de ces prestations musiciennes, la collecte de commentaires échangés parmi les spectateurs fait enfin apparaître une pluralité de critères d'appréciation, qui ne tient pas seulement aux dimensions objectivables des performances mais aussi aux conceptions que chacun se fait de la musique. Voici par exemple comment une spectatrice compare deux groupes qui ont joué le second soir :

N. (35 ans) : "Dans le cas de Hiss [un groupe de polka rock chantant en allemand], l'accent était mis sur le divertissement. Je peux très bien me les représenter dans un concert en plein air, pour un grand public. Mais pour moi, il leur manque une certaine intériorité [...]. L'ensemble Kaschu [un ensemble de jazz oriental] est pour moi un exemple de cette intériorité, de cette manière très intense de faire de la musique ensemble, de ce travail au-delà des cultures... »

Cette auditrice explique sa préférence au regard d'une certaine conception de la musique qui intègre des aspects récurrents dans l'imaginaire des musiques du monde - la liaison entre différentes cultures - mais aussi des composantes plus subjectives. D'autres auditeurs illustreront la même idée d'une liaison entre les cultures par d'autres exemples - ou contre-exemples:

Une auditrice : "Lorsque les Mongols ont chanté une chanson bavaroise, j'ai trouvé cela super. La musique du monde, c'est aussi lorsque des musiciens de différentes nationalités jouent ensemble, avec leurs instruments. Mais les musiciens de la Philharmonie avec leurs cors des Alpes (Jacaranda Ensemble), c'est pour moi aussi de la musique du monde. » 
Enkh Jarghal (musicien candidat) : "Beaucoup des groupes présents dans cette compétition ne relèvent pas des musiques du monde. Une musique du monde est une forme qui doit rassembler plusieurs cultures, faire émerger quelque chose de nouveau à partir de ses racines. »

Yann Durand (journaliste) : "Pour moi, le groupe qui a chanté un morceau d'Afrique, un morceau d'Allemagne, un morceau de Bretagne et un morceau d'Angleterre n'est pas un groupe de musiques du monde ${ }^{13}$."

Cependant, malgré ces différentes prises de position, ils passeront aussi bien souvent, à mesure que les groupes se succèdent, d'une manière d'écouter à l'autre, sans que cette hétérogénéité soit nécessairement perçue comme un problème puisqu'elle constitue une composante ordinaire dans un festival de musiques du monde. Simplement, le cadre de compétition les amène ici à s'interroger davantage sur cette catégorie, à comparer des musiques qu'ils ne comparent d'ordinaire pas et à endosser eux aussi un rôle de juge pour formuler et justifier leurs préférences ou émettre des pronostics sur les verdicts.

Que signifie en somme le fait d'écouter ensemble dans le cadre des festivals Creole? Nous avons vu que les situations de concert juxtaposent plusieurs principes de cadrage et plusieurs activités, jusqu'à susciter des questionnements sur ce qu'écouter veut dire. Elles font aussi se succéder des performances hétérogènes induisant des manières plurielles d'écouter et suscitant chacune des appréciations multiples. Le fait d'écouter ensemble signifie donc d'abord, à un degré minimal, une simple coprésence d'auditeurs, autorisant des conduites et appréciations plurielles. Larticulation entre ces différentes conduites est toutefois aussi régulée par des conventions gouvernant l'agencement d'un concert - format des 20 minutes, sonorisation, dispositif scénique - et son appréciation - conduites d'écoute, répertoire de sanctions. À cela s'ajoutent les références récurrentes à un imaginaire collectif - le thème du voyage, de la découverte du monde - et à des valeurs partagées - la tolérance, l'ouverture, la rencontre avec l'autre - qui sont constamment réaffirmées dans les programmes et prises de parole des divers acteurs présents sur la scène - la modératrice, les organisateurs, les musiciens. Enfin, cette situation donne aussi lieu à des sanctions diffuses et à des discussions en privé, qui ne rendent toutefois compte que de certains aspects des expériences. Dans la mesure où l'appréciation relève aussi du for intérieur de chaque auditeur, elle ne débouche pas nécessairement sur la formulation de jugements ou sur le débat (Schaeffer, 1996).

Le concert permet donc de combiner une hétérogénéité de performances musiciennes et de conduites d'écoute, qui n'est pas 
nécessairement ressentie comme telle par les auditeurs. Ce n'est qu'à certains moments que divers cadrages ou attentes entrent en tension. En revanche, il est un aspect qui pose toujours problème : l'évaluation. Comment comparer ces musiques incomparables?

\section{L'IMPOSSIBLE ENTENTE}

OU LA DÉLICATE PRODUCTION DES VERDICTS

Enquêtant sur Creole, j'ai été autorisée à assister aux délibérations des jurys lors des sessions régionales du premier cycle (2006-2007) et cette observation m'a amenée à nouer un dialogue avec certains des membres du collectif des organisateurs autour de ce que nous avons appelé le "problème des jurys ": comment faire en sorte que les différents jurys évaluent en fonction des mêmes critères? À l'issue du premier cycle Creole, j'ai participé à la rédaction d'un rapport d'expertise avec deux autres chercheurs qui avaient été impliqués dans le jury de la compétition Creole Rhénanie-Westphalie 2006 (Martin Greve et Michael Rappe), dans lequel nous nous sommes interrogés sur le différentiel constant entre les critères officiels de la compétition et d'autres critères implicites qui nous semblaient problématiques parce que trop mouvants ${ }^{14}$. J'ai ensuite été invitée à présenter les résultats de cette enquête lors d'une réunion du collectif des organisateurs Creole, pendant laquelle nous avons réfléchi à la composition des jurys et aux moyens de réduire la variabilité des décisions. Pour finir, j’ai été invitée à prendre part moi-même à un jury, et cette implication m'a amenée à renoncer définitivement à l'idéal de neutralité qui fondait mes premières observations pour considérer un jury pour ce qu'il est : une bataille.

Voici comment les choses se sont passées lors de la compétition Creole Rhénanie-Westphalie 2008, qui se tenait du 4 au 7 septembre dans le club du Solendo à Dortmund, pour laquelle je faisais partie du jury. Nous avions à comparer vingt et un groupes retenus par le comité de présélection et à désigner les deux groupes lauréats du prix Creole NRW 2008 admis à concourir à la finale fédérale à Berlin en septembre 2009. Soucieuse de bien faire, je m'étais préparée en écoutant la compilation envoyée deux mois plus tôt par l'organisatrice, et en notant dans mon carnet quelques pieuses résolutions tirées des leçons de mes observations antérieures - " ne pas discuter ce qui relève ou non de la Weltmusik", "c'est la qualité qui prime ", " pas de quotas ", "éviter les considérations autres que musicales ». En arrivant à Dortmund, les organisateurs nous remettent des feuillets d'évaluation sur lesquels figurent cinq critères à évaluer, sur une échelle de un à cinq. Nous 
convenons de nous retrouver à chaque pause pour discuter de ce que nous avons entendu.

Lors de la première soirée de concert, nous écoutons successivement : le Menschensinfonieorchester, un orchestre présenté dans le programme comme une "ONU musicale " offrant une " terre d'attache commune » à des personnes sans-abri ; Concopa, un quatuor de flamenco nuevo formé de deux guitaristes, un bassiste et un joueur de cajon ; l'ensemble de Daud Khan, joueur de rubab afghan qui s'est entouré pour l'occasion de sa femme, son fils et un joueur de tabla ; East affair, un quatuor de musiciens du conservatoire de Cologne qui mêle "des influences des Balkans, d'Afrique, d'Inde » avec les styles « du jazz, du funk, de la musique latine "; Fidan, un ensemble de jazz réuni autour d'une chanteuse turque. Les trois soirées suivantes amènent chacune son nouveau lot de découvertes : du folk engagé de Klaus der Geiger à la musique contemporaine chinoise de Seidenstrasse en passant par les chants rituels du Rumi Ensemble, supposant à chaque fois d'adapter les critères d'appréciation.

À cette multiplicité d'objets s'ajoute celle des perspectives amenées par les divers membres du jury. Celui-ci se compose de cinq personnes : Burkhard Hennen, fondateur du festival international de jazz de Moers, directeur du label Moers Music et amateur de musiques d'Asie; Marlon Klein, musicien d'un ensemble de world music pionnier en Allemagne - les Dissidenten -, qui a travaillé avec des musiciens de renommée internationale - Charlie Mariano, Angelique Kidjo, George Harrison - ; Jürgen Krenz, qui dirige le centre socioculturel du Goldbekhaus à Hambourg et fait partie du collectif des organisateurs de Creole ; Volker Steppat, producteur et journaliste chargé des programmes " jazz et musiques du monde " à la radio de Brême ; et moi-même, à cette époque doctorante et monitrice à l'EHESS Paris et à l'ENS de Lyon, musicienne et passionnée de musiques de Turquie. Comme pour tout jury, ces différentes personnes ont aussi été choisies parce qu'elles représentent des positions différentes au sein du champ des musiques du monde, qui ne sont pas explicitées comme telles dans le programme. Je découvre par exemple au cours de nos discussions qu'un des membres et moi ne raisonnons pas sur la base des mêmes critères. Il recherche des groupes qui puissent "jouer sur des grandes scènes", "réussir sur le marché libre ", à la différence des groupes plus réduits ou intimistes, qui ne vivent que grâce au "système des subventions ". Je cherche quant à moi à apprécier la qualité d'un point de vue artistique, en privilégiant le critère de l'innovation : critère qui n'est pas plus objectif qu'un autre mais représente une prise de position, parmi d'autres possibles, dans le champ des musiques du monde. 
Ces différences nous amènent de fait à intégrer dans nos délibérations ces arguments que j'avais pensé possible d'éviter : sur ce qui relève ou non de la Weltmusik, sur ce qui est ou non créole, et ce qui peut apparaître, pour différentes raisons, comme hors compétition. De ces arguments que j'avais précédemment critiqués, j’ai dû ici tenir compte - il eût été déplacé de les déclarer comme invalides au nom d'une vérité scientifique - et, je dois l'avouer, j'en ai aussi moi-même usé comme d'armes efficaces pour convaincre.

Lidéal de neutralité auquel nous nous accrochions dans nos rapports d'expertise n'est donc pas tenable du fait des oppositions multiples qui existent au sein du monde des musiques du monde ${ }^{15}$. Il n'est pas non plus juste dans la mesure où les prestations des musiciens impliquent chacune de prendre en compte des critères différenciés; d'effectuer des recoupements au cas par cas - que vaut ce groupe de flamenco par rapport à d'autres groupes de flamenco? Que vaut ce groupe de jazz par rapport à celui que nous avons entendu hier? Ces performances sont incomparables, mais il faut tout de même les comparer. Dans ce "tout de même " s'engouffrent des considérations qui ne tiennent pas seulement aux préférences subjectives des membres du jury mais aussi à leur aptitude à tenir compte de certaines attentes et conventions partagées. C'est ce qu'a bien compris Ben Mandelson, membre du jury de la finale du prix Creole 2007, qui décrit ainsi son travail en dissociant le registre des préférences et celui de la discussion d'experts :

Ben Mandelson : "En tant que professionnel, on est en mesure de dire : "c'est la meilleure musique dans cette catégorie" ou "c'est une musique bien faite", ou "les musiciens sont à l'aise, ils utilisent bien la scène". Nous pouvons aussi dire : "Ce n'est pas ce que j'aime, mais ils le font bien." C'est une question de goût, mais nous devons ensuite discuter et expliquer pourquoi nous aimons ceci ou cela, et c'est là qu'entre en jeu un savoir de spécialistes [...]. Dans mon travail sur des festivals, j'ai fait l'expérience que la musique ne me plait pas toujours mais que je peux tout de même décider qu'elle est bien faite et qu'elle est bien faite dans son genre. C'est toujours un mélange des deux : d'objectivité et de subjectivité. " (Entretien avec Ben Mandelson, cité d'après Greve 2007)

Lobjectivité des experts - ici définie par opposition au registre des préférences personnelles - tient à la prise en compte de certains aspects des prestations musiciennes qui leur permet de dire qu'une musique est "bien faite " et qu'elle est "bien faite en son genre ". Mais elle tient aussi à la prise en compte des attentes d'un public appréhendé à différentes échelles - le public de ce festival, 
celui des amateurs de musiques du monde, celui des jeunes générations, du "grand public ». Ces attentes qui s'imposent aux jurés parfois malgré eux, comme un ordre de choses établi, les obligent à mettre à distance leurs préférences personnelles - ce qui est souvent plus douloureux pour les musiciens et jurés novices. Elles contribuent aussi en même temps à rendre certains verdicts plus plausibles. C'est ainsi que l'incertitude tend à se réduire à mesure que l'on avance dans la compétition. Autant au moment des présélections, il est à peu près certain que deux jurys ne feraient pas les mêmes choix ${ }^{16}$, autant au moment des finales, les verdicts des jurys deviennent plus prédictibles. Ils éliront alors généralement des groupes dont la musique est "bien faite ", mais aussi dont les performances mettent en avant certaines marques d'origine - langue, costumes, instruments -, relient des genres supposés distants - le jazz et le maqam d'Irak, la musique mongole et la chanson bavaroise - et sont susceptibles de circuler sur la scène internationale, ce qui tend à exclure les groupes chantant en allemand. Ils tendront, à l'inverse, à exclure des groupes trop traditionnels, classiques ou expérimentaux. Et ces verdicts susciteront bien entendu aussi, de manière assez constante, des critiques autant parmi les spectateurs que parmi les organisateurs des festivals.

Tout au long du festival cependant, ceux-ci n'auront cessé de répéter que Creole n'est pas seulement une compétition. Et c'est notamment au moment de l'annonce des verdicts qu'ils veilleront à relativiser cette concurrence par de multiples réparations symboliques : en félicitant tous les candidats et en soulignant la difficulté de la tâche des jurys - " tous auraient mérité un prix spécial ", dira Christoph Borkowsky Akbar, président du jury du prix Creole 2009 lors de la remise des prix -, en organisant d'autres moments de rencontre - une session de danse animée par un DJ, une cérémonie de clôture associant tous les musiciens, un brunch offert le lendemain, et en rappelant constamment l'importance d'être solidaires dans la défense des musiques du monde de valeurs qui vont bien au-delà de la musique.

ambiguë qui a cours dans nos sociétés et dans certains secteurs de l'activité musicienne : "l'écoute du monde ", que j’ai ici questionnée à partir d'un cas singulier. Décrivant certains aspects du processus de réalisation des festivals Creole, j'ai d'abord expliqué combien le repérage et la sélection de "musiques du monde " s'effectuent sur la base de critères multidimensionnels et 
mouvants. Enquêtant sur le moment des concerts, j'ai montré que ces situations juxtaposent plusieurs principes de cadrages qui peuvent parfois entrer en tension, jusqu'à susciter des questionnements sur ce qu'écouter veut dire. Décrivant les conduites et commentaires de certains auditeurs, j'ai mis en évidence la variété des modalités d'appréciation mais aussi la possibilité de passer de l'une à l'autre sans que cette hétérogénéité soit nécessairement perçue comme problématique. Enfin, la participation aux délibérations a fait apparaître la pluralité des points de vue des divers experts mobilisés dans ces jurys et, au-delà de Creole, la prégnance de lignes de frontière - musiques sérieuses ou de divertissement, traditionnelles ou modernes, élitistes ou populaires - autour desquelles s'organisent des prises de position.

Ce faisant, ce parcours m'a amenée à mobiliser différentes acceptions de l'adverbe "ensemble ", que je me dois à présent d'expliciter. Lorsque l'on dit d'une pluralité d'auditeurs qu'ils sont ensemble lors d'une soirée de concert, on fait d'abord référence à la simultanéité de leur présence. Mais il arrive aussi que cette rencontre alimente parmi les participants le sentiment d'une communauté d'attentes et de valeurs, qui s'exprimera alors à travers un "nous " à échelle variable - "nous les organisateurs", "nous les amateurs de musiques du monde", "nous les Allemands ", "nous citoyens du monde "-, dont il importe de souligner la solidarité. C'est d'autre chose qu'il s'agit lorsque l'on entreprend de décrire ce que font ensemble des personnes réunies le temps d'un concert : puisque pour faire quelque chose, elles n'ont pas besoin de penser ni de faire la même chose. Un festival associe, ainsi qu'on a pu le voir, différentes activités, manières d'écouter et appréciations. En revanche, quand des personnes sont placées en situation de devoir délibérer sur des performances musicales, leur discussion porte sur certains cas qu'ils doivent comparer en tenant compte de certains critères mais aussi de la singularité de chaque cas. Or, cette hétérogénéité particulièrement forte des objets contribue probablement à expliquer les difficultés que rencontrent les acteurs des musiques du monde à s'associer. Il existe un champ professionnel des musiques du monde qui a pu se constituer à travers des rassemblements à large échelle - des marchés ou rencontres internationales comme le World Music Expo, Babelmed à Marseille -, mais cette association est toujours plus délicate à plus petite échelle, lorsqu'il s'agit de mobiliser divers acteurs d'une ville ou région ${ }^{17}$ ayant à voir avec les musiques du monde - festivals, agences de production, conservatoires et écoles de musique, musées, lieux de concert, centres sociaux, instituts de recherche parce que cette rencontre fait alors ressortir des disparités entre différents horizons institutionnels et régimes d'action. Or, dans 
ce monde des musiques du monde où la tonalité dominante est celle d'un enthousiasme militant et où il est rare de voir s'exprimer en public des critiques sur la qualité de telle ou telle musique, il n'est pas anodin que les disputes les plus vives surgissent lorsque des personnes se voient placées en situation de devoir écouter et évaluer ensemble de la musique.

T. B. $-L$.

Université Jean Monnet de Saint-Étienne (Cierec) École des hautes études en sciences sociales (centre Georg Simmel)

Manuscrit reçu le : 31 juillet 2014

Version révisée reçue le : 25 janvier 2015

Article accepté pour publication le : 06 février 2015 
NOTES

1. Outre les festivals Creole sur lesquels se centre cet article : le Festival de l'imaginaire à Paris (2007-2008), le festival Villes des musiques du monde (2009), le Womex (2007), le TFF Rudolstadt (2007), le Carnaval des cultures de Berlin (2006).

2. Selon le sous-titre de la manifestation, remplacé en 2011 par " musique globale d'Allemagne" puis en anglais "Global Music Contest ".

3. Notons au passage que cette formulation ne va pas sans deux postulats: d'une part, que ces pratiques relèvent bien de "la musique ", d'autre part qu'il existerait un domaine où la musique ne serait que de la musique et où l'on ne ferait donc que l'écouter pour elle-même.

4. Ces différences sont telles qu'il est difficile de dire ce qui est commun entre tous ces festivals - par exemple : le Festival de l'imaginaire (Paris) qui se centre sur des traditions rituelles et spectaculaires peu connues du public; Villes des musiques du monde (Seine-SaintDenis) qui programme des artistes renommés mais aussi de nombreux ateliers et concerts avec des musiciens amateurs ; le TFF Rudolstadt, un festival qui attire des milliers de spectateurs dans une petite ville de l'est de l'Allemagne.

5. Les comités de présélection sont composés généralement de "locaux" (habitants de la ville ou de la région), les jurys des festivals régionaux incluent au moins un représentant d'une autre région.

6. Une enquête par questionnaire réalisée lors de la finale du prix Creole 2009, qui se tenait dans la salle du Huxley's Neue Welt à Berlin, a abouti aux résultats suivants : sur 103 questionnaires rendus, $36 \%$ des spectateurs étaient non berlinois (pour beaucoup, venus avec des musiciens candidats) et une part significative (23\%) y retrouvait des collègues.
7. Outre les débats fréquemment organisés en parallèle des festivals, la finale du prix 2011 a ainsi été assortie d'une rencontre professionnelle pensée sur le modèle du World Music Expo.

8. Au sens de Ruwen Ogien, qui désigne par cette expression diverses formes d'appréciations (commentaires, rires, sarcasmes...) manifestant un " ordre social informel " par opposition aux sanctions "légales " (que représenteraient ici les verdicts des jurys).

9. Relisant mes notes de terrain, je suis frappée de la récurrence de remarques sur le volume alors que dans mes entretiens, ces aspects sont peu thématisés.

10. Parmi les spectateurs qui ont répondu au questionnaire lors de la finale du prix Creole 2009, 55 \% avaient plus de 40 ans. Les sondages que j'ai effectués lors d'une session régionale donnaient une proportion plus importante de personnes de moins de 40 ans (3/4).

11. Le développement croissant de festivals a eu pour effet de promouvoir une injonction à danser, contre laquelle s'insurgeait le critique Jan Reichow lors d'une émission diffusée en 2004 sur WDR 3 : "Il n’y a pas qu'en Occident que l'on montre sa culture en restant assis. Dans bien des parties du monde, le fait d'écouter ou de discuter en bougeant de manière permanente des hanches est considéré comme peu pertinent, surtout lorsque l'on rencontre des personnes d'autres cultures. Seule l'idéologie à la mode du Multi-Kulti a développé cette obligation énervante de danser. » (Émission Musikpassagen du 20 octobre 2004, texte en ligne sur www.janreichow.de)

12. Les groupes ne reçoivent pas de gages pour leur participation mais y gagnent d'autres avantages (publicité, enregistrements, photos et vidéos). Les lauréats des sessions régionales et fédérales reçoivent des prix allant de 1000 à 5000 euros. 
13. Entretiens réalisés par une équipe de la Musikhochschule de Cologne dont je faisais partie, cités d'après Greve 2007.

14. Parmi ces critères : celui de l'appartenance au genre "musiques du monde " ou l'idée qu'il faudrait choisir trois lauréats différents.

15. Il existe bien un champ des musiques du monde structuré par des rassemblements professionnels, des experts du genre et de médias spécialisés, mais ce champ ne rassemble qu'une partie du "monde" des musiques du monde dans lequel j'inclus aussi d'autres acteurs mobilisant ces musiques (conservatoires, lieux de concerts classiques, musées et archives sonores, organisations internationales).

16. L'expérience a été tentée par Martin Greve et Michael Rappe lors d'un séminaire à la Musikhochschule de Cologne pour lequel ils avaient confié l'évaluation de vingt dossiers à cinq groupes d'étudiants, dont les verdicts ont été très différents.

17. C'est ce que montre l'évolution de Creole qui n'a pas débouché, ainsi que le souhaitaient certains membres du collectif des organisateurs, sur la création d'une association fédérale. C'est aussi ce que montrent les observations que j'ai pu mener en France sur les États généraux de Zone Franche, le forum des musiques traditionnelles de Rhône-Alpes ou le collectif musiques et danses du monde en Île-de-France.

\section{RÉFÉRENCES BIBLIOGRAPHIQUES}

Aubert (Laurent). 2001. La Musique de l'autre: Les nouveaux défis de l'ethnomusicologie. Genève : Georg éditeur.

Bachir-Loopuyt (Talia). 2013. Une musique du monde faite en Allemagne? Les compétitions Creole et l'idéal d'une société plurielle dans l'Allemagne d'aujourd'bui. Th. Doc. : EHESS, Paris. Publication en ligne: https://tel.archives-ouvertes.fr/tel00927409

Becker (Howard). 1988. Les Mondes de l'art. Paris : Flammarion.

Bensignor (François). 2002. Les Musiques du monde. Paris : Larousse.

Bohlman (Philip). 2002. World Music: A Very Short Introduction. Oxford : Oxford University Press.

Broughton (Simon) \& Burton (Kim). 1994. World Music. London : Rough Guides.

Cefaï (Daniel) \& Terzi (Cédric) (sous la direction de). 2012. L'Expérience des problèmes publics. Paris : Éd. de l'EHESS, coll. "Raisons Pratiques ", 22.

Collectif. 2001. Le Spectacle des autres : Questions d'ethnoscénologie 2. Internationale de l'Imaginaire, 15, Arles : Actes Sud.

Descombes (Vincent). 1996. Les Institutions du sens. Paris : Éd. de Minuit.

Dodier (Nicolas). 1993. "Les appuis conventionnels de l'action : Éléments de pragmatique sociologique", Réseaux, 11(62), p. 63-85.

Fabiani (Jean-Louis). 2012. "Festivals, local and global : critical interventions and the cultural public sphere ", p. 92-107, in Festivals and the Cultural Public Sphere / dirigé par Liana Giorgi, Monica Sassatelli et Gerard Delanty. Londres : Routledge.

Favret-Saada (Jeanne). 1977. Les Mots, la mort, les sorts. Paris : Gallimard.

Fornel (Michel de) \& Quéré (Louis) (sous la direction de). 1999. La Logique des situations: Nouveaux regards sur l'écologie des activités sociales. Paris : Éd. de l'EHESS, coll. "Raisons pratiques ", 10. 
Goffman (Erving).1991. Les Cadres de l'expérience, Paris : Éd. de Minuit.

Greve (Martin) et al. 2007. Der Bundeswettbewerb Creole 2007: Dokumentation und Empfeblungen, Hochschule für Musik, Cologne. [Rapport d'expertise non publié.]

Guilbault (Jocelyne). 1993. Zouk: World Music in the West Indies. Chicago / Londres : Chicago University Press.

Joas (Hans). 1992. Die Kreativität des Handelns. Francfort : Suhrkamp.

Koselleck (Reinhart). 1990 [1977]. Le Futur passé : Contribution à la sémantique des temps bistoriques. Paris : Éd. de l'EHESS.

Laborde (Denis). 1998. Musiques à l'école. Paris : Bertrand-Lacoste.

Maisonneuve (Sophie). 2009. "L'expérience festivalière : Dispositifs esthétiques et arts de faire advenir le goût ", p. 85-115, in Écologie sociale de l'oreille: Enquêtes sur l'expérience musicale / sous la direction d'Anthony Pecqueux \& Olivier Roueff. Paris : EHESS.

Négrier (Emmanuel). 2014. Les Musiques du monde et leur(s) public(s). Marseille : Le Mot et le Reste.

Ogien (Ruwen). 1990. "Sanctions diffuses : Sarcasmes, rires, mépris", Revue française de sociologie, 31(4), p. 591-607.

Olivier (Emmanuelle) (sous la direction de). 2014. "Composer avec le monde", Volume! 10(2).

Pecqueux (Anthony). 2003. "Indifférence, attention, latéralité : Ethnographie d'un concert de rap", p. 319-337, in Le Public en action: Usages et limites de la notion d'espace public en sciences sociales / sous la direction de Claudia Barril, Marion Carrel, Juan-Carlos Guerrero \& Alicia Marquez. Paris : L'Harmattan.

Pecqueux (Anthony) \& Roueff (Olivier) (sous la direction de). 2009. Écologie sociale de l'oreille: Enquêtes sur l'expérience musicale. Paris : Éd. de l'EHESS.

Peterson (Richard A.). 2004. "Le passage à des goûts omnivores : Notions, faits et perspectives ", Sociologie et Sociétés, 36(1), p. 145-164.
Schaeffer (Jean-Marie). 1996. Les Célibataires de l'art: Pour une esthétique sans mythe. Paris : Gallimard.

White (Bob) (sous la direction de). 2012. Music and Globalization : Critical Encounters. Bloomington : Indiana University Press.

Zemp (Hugo). 1996. "The/An ethnomusicologist and the music business ", Yearbook for Traditional Music, 28, p. 36-56. 


\section{RÉSUMÉS}

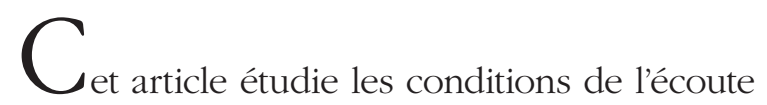

dans un festival de musiques du monde en variant les points de vue et perspectives. Plutôt que de chercher à caractériser un idéal type ("le festival de musiques du monde»), je pars ici d'un cas singulier (des compétitions intitulées Creole. Prixpour les musiques du monde d'Allemagne) que j'appréhende à partir des situations d'épreuve afin de rendre compte de la superposition d'attentes et de cadres pluriels. Au-delà de la place que lui assignent les études de réception, j'analyse l'écoute comme une pratique collective impliquant une pluralité d'acteurs (organisateurs, experts des comités de sélection et des jurys, musiciens, spectateurs) qui interagissent selon des conditions variables d'une étape à l'autre de la réalisation du festival. La fabrique de la programmation, le moment public des concerts et celui, plus confidentiel, des délibérations d'un jury sont ici envisagés comme autant d'occasions de "faire ensemble "supposant, à chaque fois, de réajuster les modalités de l'écoute et de l'appréciation.

Titre : Être ensemble, écouter, évaluer les musiques du monde en festival

Mots-clés : Musiques du monde, festival, concert, écoute, appréciation.

\section{$T_{b}$ \\ his article studies the conditions of music} listening at a world music festival from diverse viewpoints and perspectives. Rather than attempting to characterize the ideal or typical "world music festival", I have based my study on a single example: the Creole World Music from Germany contest. In exploring the competition phases, I seek to understand how expectations and multiple settings merge. Above and beyond the role of perception on which research generally focuses, I analyze listening as a collective practice involving multiple players (organizers, experts from selection committees and judging panels, musicians, spectators) who interact in varying conditions throughout the different phases of the festival organization. The process of putting the program together, the concert performances in public, and the judges' deliberations behind the scenes are treated in this study as opportunities for "doing together" that require, for each, a readjustment of the manner in which the music is listened to and assessed.

Title: Being together, listening, assessing world music in a festival

Keywords: World music, festival, concert, listening, assessment. 


\section{$\mathrm{E}_{\text {ste artículo estudia las condiciones de escu- }}$}

cha en un festival de músicas del mundo, cambiando los puntos de vista y las perspectivas. En lugar de caracterizar un ideal tipo - "el festival de músicas del mundo "-, aquí salgo de un caso singular - de las competiciones llamadas criollas. Premio para las músicas del mundo de Alemania - que percibo a partir de las situaciones de prueba, para describir la superposición de expectativas y de marcos múltiples. Más allá del lugar que le asignan los estudios de recepción, analizo la escucha como una práctica colectiva que implica una multiplicidad de actores (organizadores, expertos de las comisiones de selección y de los jurados, músicos, espectadores) que interactúan según las condiciones variables de una a otra etapa de la realización del festival. La preparación de la programación, el momento público de los conciertos y, más confidencial, el de las deliberaciones de un jurado, se consideran aquí como tantas oportunidades para " hacer juntos " lo que supone cada vez, reajustar las modalidades de la escucha y de la apreciación.

Título : Estar juntos, escuchar, evaluar las músicas del mundo en festival

Palabras claves : Músicas del mundo, festival, concierto, escucha, apreciación. 